\title{
Metastatic malignant struma ovarii with coexistence of Hashimoto's thyroiditis
}

\author{
Marco Russo',*, llenia Marturano2,*, Romilda Masucci³, Melania Caruso4, \\ Maria Concetta Fornito5, Dario Tumino², Martina Tavarelli², Sebastiano Squatrito² \\ and Gabriella Pellegriti6 \\ ${ }^{1}$ Endocrinology, Department of Clinical and Experimental Medicine, University of Messina, Messina, \\ Italy, ${ }^{2}$ Endocrinology, Department of Clinical and Experimental Medicine, University of Catania, \\ Garibaldi-Nesima Hospital, Catania, Italy, ${ }^{3}$ Surgical Oncology, ${ }^{4}$ Gynecology and Obstetrics, \\ ${ }^{5}$ Nuclear Medicine and ${ }^{6}$ Endocrinology, Garibaldi-Nesima Hospital, Catania, Italy \\ *(M Russo and I Marturano contributed equally to this work)
}

\author{
Correspondence \\ should be addressed \\ to G Pellegriti \\ Email \\ g.pellegriti@unict.it
}

\section{Summary}

Struma ovarii is a rare ovarian teratoma characterized by the presence of thyroid tissue as the major component. Malignant transformation of the thyroidal component (malignant struma ovarii) has been reported in approximately $5 \%$ of struma ovarii. The management and follow-up of this unusual disease remain controversial. We report the case of a woman with a history of autoimmune thyroiditis and a previous resection of a benign struma ovarii that underwent hystero-annexiectomy for malignant struma ovarii with multiple papillary thyroid cancer foci and peritoneal involvement. Total thyroidectomy and subsequent radioiodine treatment lead to complete disease remission after 104 months of follow-up. The diagnosis and natural progression of malignant struma ovarii are difficult to discern, and relapses can occur several years after diagnosis. A multidisciplinary approach is mandatory; after surgical excision of malignant struma, thyroidectomy in combination with ${ }^{131}$ | therapy should be considered after risk stratification in accordance with a standard approach in differentiated thyroid cancer patients.

\section{Learning points:}

- Malignant struma ovarii is a rare disease; diagnosis is difficult and management is not well defined.

- Predominant sites of metastasis are adjacent pelvic structures.

- Thyroidectomy and ${ }^{131}$ il therapy should be considered after risk stratification in accordance with standard approaches in DTC patients.

\section{Background}

Struma ovarii is a rare ovarian teratoma in which thyroid tissue accounts for more than $50 \%$ of the teratoma mass. Up to $20 \%$ of teratomas contain thyroid tissue, but struma ovarii is only diagnosed when the thyroid tissue accounts for $50 \%$ or more of the tumor cellular component. Struma ovarii represents only approximately $3 \%$ of mature teratomas and $1 \%$ of all ovarian tumors (1).



Malignant transformation is rare, and the differential diagnosis between benign and malignant struma ovarii may be difficult.

Moreover, the true incidence is not easy to establish because of the rarity of this neoplasm, but histological findings of thyroid carcinoma are observed in approximately $5 \%$ of struma ovarii. Metastatic spread

(C) 2016 The authors http://www.edmcasereports.com Published by Bioscientifica Ltd 
ranges from 4 to $23 \%$ of cases, depending on the series reported in the literature, and adjacent pelvic structures are typically involved (1) (2).

We describe a case of malignant struma ovarii with widespread metastastic dissemination and a good response to ${ }^{131}$ iodine (131I) treatment.

\section{Case presentation}

In 1999, following the onset of abdominal pain, a 27-yearold woman underwent medical examinations that revealed evidence of an $8 \mathrm{~cm}$ left ovarian mass with both solid and cystic components. After the complete laparoscopic mass resection, histopathological examination revealed a benign struma ovarii. For many years after surgery, the patient complained of tachycardia with associated asthenia and periodically presented to her endocrinologist. TSH and thyroid hormone measurements exhibited slight subclinical hyperthyroidism with high antithyroglobulin (AAT) and antiperoxidase (AbTPO) antibody levels while TSH receptor antibodies (TRAb) were not detectable. Thyroid ultrasound was consistent with autoimmune thyroiditis. Subsequent clinical examinations revealed a stable condition until 2006 when following the onset of frequent abdominal cramps and pain, the patient underwent an intravaginal pelvic ultrasound that revealed a 1.9 and a $2.9 \mathrm{~cm}$ cyst in the right ovary and in the pouch of Douglas, respectively.

\section{Investigation}

Tumor markers (CEA, Ca-19.9, Ca 15.3, Ca 125, alpha FP) were all negative. Due to the declining clinical condition and upon suspicion of appendix inflammation, a laparoscopy intervention was performed. A $0.7 \mathrm{~cm}$ mesenteriolum node was found in the appendix. On pathological examination, the appendicular tissue was determined to be a follicular variant of papillary thyroid cancer. The patient was referred to our endocrinology clinic for further management. Thyroid function was assessed: serum TSH $(1.8 \mu \mathrm{U} / \mathrm{mL}$; reference range: $0.34-4.94 \mu \mathrm{U} / \mathrm{mL})$, FT4 and FT3 values were in the normal range, serum AAT and AbTPO antibodies were elevated $(1475 \mathrm{U} / \mathrm{mL}$ and $297 \mathrm{U} / \mathrm{mL}$; reference range: 0-34 U/mL and 0-12 $\mathrm{U} / \mathrm{mL}$, respectively), and the serum thyroglobulin ( $\mathrm{Tg}$ ) concentration was $4.8 \mathrm{ng} / \mathrm{mL}$ (reference range: $1.1-130 \mathrm{ng} / \mathrm{mL}$ ). Thyroid ultrasonography revealed a hypo-echogenic parenchyma, indicating chronic thyroiditis. Magnetic resonance of the abdomen revealed a $2 \mathrm{~cm}$ right annex mass with irregular margins. The patient subsequently underwent hysterectomy and bilateral annexiectomy, pelvic-selective lymphadenectomy, and total thyroidectomy. Pathological examination revealed a bilateral struma ovarii with foci of multiple follicular variants of well-differentiated papillary thyroid carcinoma; similar foci were found in the left periophorogenous area, in the prevesical peritoneum, in the pouch of Douglas, and within the omentum. No neoplastic involvement was found in the abdominal lymph nodes or in the thyroid parenchyma that exhibited signs of thyroiditis.

\section{Treatment}

The patient started L-thyroxine therapy after total thyroidectomy and 3 months after surgery underwent radioiodine treatment with $3.7 \mathrm{GBq}{ }^{131}$ I after L-thyroxine withdrawal. Post-treatment whole-body scan (WBS) revealed a focus of ${ }^{131}$ I uptake in the left subcricoid paramedian area, several foci in the paravertebral abdominal area, and two foci in the right and left hypochondrium, respectively (Fig. 1A). Tg levels were undetectable with elevated AAT levels $(1658 \mathrm{U} / \mathrm{mL})$. The patient underwent CT of the chest, abdomen, and pelvis, which did not reveal structural persistent disease; bone scan, ultrasonography, and CT of the neck were normal.

\section{Outcome and follow-up}

During the following 12 months, due to the high AAT levels and the pathological ${ }^{131} \mathrm{I}$ uptake, the patient underwent another treatment with $3.7 \mathrm{GBq}{ }^{131} \mathrm{I}$, and the WBS revealed feeble ${ }^{131}$ I uptake in left hypochondrium and hypogastrium. Tg levels were undetectable with


T.B 1131 POST100mC

T.BI131POST100m.Cs

Figure 1

Image of the first (A) and last (B) whole-body scan after the administration of $3.7 \mathrm{GBq}$ of ${ }^{131}$. 
progressive reduction in AAT antibodies levels $(507 \mathrm{U} / \mathrm{mL})$. A third treatment with $3.7 \mathrm{GBq}$ of ${ }^{131} \mathrm{I}$ (cumulative dose 11.1 GBq) was performed 12 months later with a negative post-therapy WBS (Fig. 1B) and a further reduction in serum AAT antibodies $(170 \mathrm{U} / \mathrm{mL})$. At the last control visit (104 months from diagnosis), the patient, on L-thyroxine therapy, was feeling well without abdominal discomfort, serum Tg was undetectable, and AAT antibodies exhibited a further decrease $(45 \mathrm{U} / \mathrm{mL})$; no evidence of disease was present.

\section{Discussion}

Malignant struma ovarii is a rare ovarian tumor and many diagnostic and therapeutic aspects are debated because of its rarity. Previous studies reported that malignant struma ovarii is more common in the fourth decade of life and that the majority of patients are asymptomatic or complain of nonspecific symptoms (3). At the time of diagnosis, the most common symptoms are pelvic pain and the appearance of a pelvic mass; less frequently, ascites, hyperthyroidism (described in approximately $5-8 \%$ of all struma ovarii), menstrual irregularities, or symptoms caused by metastatic spread are reported (4). Distant metastasis has been reported to be uncommon, although some larger series indicate higher frequency (23\%), mainly due to widespread tumors in adjacent pelvic structures (peritoneum, omentum, mesentery, fallopian tubes, and contralateral ovary) and less frequently to the lungs, bone, liver, and brain (2) (4).

Malignant struma ovarii is diagnosed when classical histological features of thyroid carcinoma are observed in struma ovarii, including the presence of ground glass nuclei, cellular atypia, nuclear grooves, and inclusion bodies (5). The presence of distant metastasis supports the diagnosis of malignancy. The most common histology is papillary carcinoma followed by follicular carcinoma. Although very rare, ovarian metastasis of thyroid carcinoma should be differentiated from malignant struma ovarii by the absence of features of teratoma and the concomitant presence of thyroidal primary lesion (6). The histological features of malignancy in struma ovarii, however, often do not correspond to clinically malignant behavior, which remains enigmatic.

Recurrence rates are variable and depending on the series, de Simone and coworkers reported a rate of $35 \%$ and a median time to recurrence of 4 years (7). Overall survival is, however, excellent and Goffredo and coworkers reported OS at 5, 10, and 20 years of 96.7, 94.3, and $84.9 \%$, respectively (3).
The optimal treatment and management of patients with malignant struma ovarii have not been well defined. Initial pelvic surgery consists of total hysterectomy with bilateral salpingo-oophorectomy, omentectomy, peritoneal washings, and lymph nodes sampling; in young women, to preserve fertility, unilateral oophorectomy is advocated (7). After surgical removal of the ovarian mass and histological diagnosis of malignant struma ovarii, some authors suggest thyroidectomy and ${ }^{131}$ I therapy with ablative dose (4) (7). Thyroidectomy enables effective ${ }^{131}$ I ablative treatment and facilitates the subsequent followup by enhancing serum Tg sensibility. Furthermore, thyroidectomy permits the exclusion of a primitive thyroid cancer. Post-therapeutic WBS is helpful to localize metastatic lesions. ${ }^{131} \mathrm{I}$ is effective in the treatment of recurrent disease and led to a complete remission in our patient. A risk stratification of malignant struma ovarii similar to that used in differentiated thyroid cancer might help to determine appropriate postoperative treatment. In this regard, Yassa and others suggested L-thyroxine therapy and periodic pelvic imaging with Tg measurement in lowrisk patients only and strongly recommend thyroidectomy and ${ }^{131}$ I therapy in high-risk patients (4). Moreover, when extraovarian disease is present, 131I should be the first-line therapy; external radiation treatment and chemotherapy should be used only in selected cases. In our patient, 131I therapy had beneficial effects on extraovarian disease; WBS was negative at the last treatment, and serum AAT antibodies significantly decreased, which may reflect a response to ${ }^{131}$ I therapy.

Finally, we can only speculate that highly elevated AAT and autoimmune thyroiditis might be a consequence of immune response to extraovarian cancer dissemination or of an autoimmune process that first started in the struma ovarii and caused a secondary thyroiditis. Hashimoto's thyroiditis with a struma ovarii has been described in a few case reports (8) (9) and in a report describing a patient diagnosed with autoimmune thyroiditis in malignant struma ovarii (9). Coexistence of thyroiditis and papillary thyroid cancer is not uncommon, and it has been hypothesized a link between the two disease; this coincidence implies the use of AAT during follow-up as a surrogate marker because of the interference with $\mathrm{Tg}$ assay (10). Moreover, some evidences indicate that the presence of autoimmune thyroiditis may determine a better outcome of thyroid cancer (10).

In conclusion, our case demonstrates the difficulty in discerning the natural progression of this disease, that relapse can occur several years after diagnosis and the need for a multidisciplinary approach. In our 
patient, 131I therapy effectively treated the metastatic disease, and we believe that this therapeutic tool in combination with thyroidectomy should be always considered after proper risk stratification in accordance with standard approaches in differentiated thyroid cancer patients.

\section{Declaration of interest}

The authors declare that there is no conflict of interest that could be perceived as prejudicing the impartiality of the research reported.

\section{Funding}

This research did not receive any specific grant from any funding agency in the public, commercial, or not-for-profit sector.

\section{Patient consent}

Written informed consent was obtained from the patient for publication of this Case report and any accompanying images.

\section{Author contribution statement}

$\mathrm{R} \mathrm{M}$, I M, D T, M T treated the patient, gathered data, and drafted the manuscript; $M R, M C$ treated the patient, carried out the surgery, and critically reviewed the manuscript; M C F treated the patient and critically reviewed the manuscript; S S critically reviewed the manuscript and gave the final approval of the version to be published; G P treated the patient, conceptualized the case report, gathered data, and critically reviewed the manuscript.

\section{References}

1 Talerman A. Germ cell tumors of the ovary. In Blaustein's Pathology of the Female Genital Tract, edn 3, pp 659-721. Ed RL Kurman. New York, NY, USA: Springer-Verlag, 2011. (doi:10.1007/978-1-4419-0489-8_16)

2 Makani S, Kim W \& Gaba AR 2004 Struma Ovarii with a focus of papillary thyroid cancer: a case report and review of the literature. Gynecologic Oncology 94 835-839. (doi:10.1016/j.ygyno.2004.06.003)

3 Goffredo P, Sawka AM, Pura J, Adam MA, Roman SA \& Sosa JA 2015 Malignant struma ovarii: a population-level analysis of a large series of 68 patients. Thyroid 25 211-215. (doi:10.1089/thy.2014.0328)

4 Yassa L, Sadow P \& Marqusee E 2008 Malignant struma ovarii. Nature Clinical Practice Endocrinology \& Metabolism 20084 469-472. (doi:10.1038/ncpendmet0887)

5 Devaney K, Snyder R, Norris HJ \& Tavassoli FA 1993 Proliferative and histologically malignant struma ovarii: a clinicopathologic study of 54 cases. International Journal of Gynecologic Pathology 12 333-343. (doi:10.1097/00004347-199310000-00008)

6 Brogioni S, Viacava P, Tomisti L, Martino E \& Macchia E 2007 A special case of bilateral ovarian metastases in a woman with papillary carcinoma of the thyroid. Experimental and Clinical Endocrinology \& Diabetes 115 397-400. (doi:10.1055/s-2007-973853)

7 DeSimone CP, Lele SM \& Modesitt SC 2007 Malignant struma ovarii: a case report and analysis of cases reported in the literature with focus on survival and I131 therapy. Gynecologic Oncology 89 543-548. (doi:10.1016/S0090-8258(03)00141-0)

8 Bozkurt NC, Karbek B, Ozkaya EC, Cakal E \& Delibaşi T 2011 Struma ovarii presenting with Hashimoto's thyroiditis: a case report. Journal of Medical Case Reports 20115 572. (doi:10.1186/1752-1947-5-572)

9 Doldi N, Taccagni GL, Bassan M, Frigerio L, Mangili G, Jansen AM \& Ferrari A 1998 Hashimoto's disease in a papillary carcinoma of the thyroid originating in a teratoma of the ovary (malignant struma ovarii). Gynecological Endocrinology 12 41-42. (doi:10.3109/09513599809024969)

10 Feldt-Rasmussen U \& Rasmussen AK 2010 Autoimmunity in differentiated thyroid cancer: Significance and related clinical problems. Hormones 9 109-117. (doi:10.14310/horm.2002.1149)

Received in final form 11 March 2016

Accepted 27 April 2016 